\title{
Optimization of frequency-doubled Er-doped fiber laser for miniature multiphoton endoscopy
}

\author{
Lin Huang \\ Xin Zhou \\ Shuo Tang
}




\title{
Optimization of frequency-doubled Er-doped fiber laser for miniature multiphoton endoscopy
}

\author{
Lin Huang, Xin Zhou, and Shuo Tang* \\ University of British Columbia, Department of Electrical and Computer Engineering, Vancouver, Canada
}

\begin{abstract}
Frequency-doubled femtosecond Er-doped fiber laser is a low-cost and portable excitation source suitable for multiphoton endoscopy. The frequency-doubled wavelength at $780 \mathrm{~nm}$ is used to excite the intrinsic fluorescence signal. The frequency-doubling with a periodically poled $\mathrm{MgO}: \mathrm{LiNbO}_{3}(\mathrm{PPLN})$ is integrated in the distal end of the imaging head to achieve fiber connection. The imaging speed is further improved by optimizing the excitation laser source. A 0.3-mm length of PPLN crystal is selected and the Er-doped fiber laser is manipulated to match its bandwidth with the acceptance bandwidth of the PPLN. Through this optimization, a reduced pulsewidth of $80 \mathrm{fs}$ of the frequency-doubled pulse is achieved. All-fiber dispersion compensation and pulse compression by single mode fiber is conducted, which makes the fiber laser directly fiber-coupled to the imaging head. An imaging speed of 4 frames/s is demonstrated on ex vivo imaging of unstained biological tissues, which is 10 times faster than our previous study using a 1-mm-long PPLN. The results show that miniature multiphoton endoscopy using frequency-doubled Er-doped fiber laser has great potential for clinical applications. @ The Authors. Published by SPIE under a Creative Commons Attribution 3.0 Unported License. Distribution or reproduction of this work in whole or in part requires full attribution of the original publication, including its DOI. [DOI: 10.1117/1.JBO.23.12.126503]
\end{abstract}

Keywords: multiphoton microscopy; endoscopic imaging; laser scanning microscopy.

Paper 180332RR received Jun. 5, 2018; accepted for publication Nov. 26, 2018; published online Dec. 21, 2018.

\section{Introduction}

Multiphoton microscopy (MPM) is an effective and noninvasive imaging tool that enables the visualization of living tissues and organs. ${ }^{1,2}$ In MPM, near-infrared femtosecond pulsed lasers are used to excite nonlinear contrast signals such as two-photon excitation fluorescence (TPEF) and second harmonic generation (SHG) ${ }^{3-5}$ In order to utilize MPM in clinical applications, miniature MPM endoscopy needs to be developed. In MPM endoscopy, the excitation laser pulse and the MPM signals can be guided through flexible optical fiber, and thus various tissue locations can be conveniently imaged via a miniature probe. ${ }^{6-9}$ To date, several different groups proposed MPM endoscopy systems and applied them for different applications. ${ }^{10-16}$

Delivering femtosecond pulses in optical fiber is challenging due to the temporal and spectral broadening from group velocity dispersion (GVD) and self-phase modulation (SPM). Huland et $\mathrm{al}^{8}{ }^{8}$ developed an MPM endoscopy system where the pulse from a Ti:Sapphire laser was delivered by a hollow core photonic bandgap fiber (PBF). In vivo imaging was conducted on unstained tissue in anesthetized rats at 4 frames/s. In PBF, light propagates in an air core where GVD is minimized. However, PBF has limited bandwidth and cannot deliver ultrashort pulses. Helmchen et al. ${ }^{6}$ reported a miniature MPM endoscopy for imaging the brain of freely moving mice. Femtosecond pulse from a Ti:Sapphire laser passed through a grating pair for precompensating GVD, and then was coupled into a single mode fiber (SMF) for delivery. The system had a relatively bulky prototype and the imaging speed was only 0.5 frames/s. An improved linear and nonlinear pre-compensation scheme was also developed, ${ }^{17,18}$ where the pulse was first spectrally broadened by a piece of SMF, then temporally stretched by a free-space dispersive line (gratings or prisms), and finally the pulse was temporally and spectrally recompressed in a delivery fiber. Using

*Address all correspondence to Shuo Tang, E-mail: tang@ece.ubc.ca this scheme, Ducourthial et al. ${ }^{15}$ delivered 39 fs pulses at $20 \mathrm{~mW}$ average power over 5-m-long double cladding photonic crystal fiber. Liang et al. ${ }^{19}$ delivered 100 -fs pulses at $45-\mathrm{mW}$ average power over $0.8-\mathrm{m}$-long double cladding fiber. The limitation of this scheme is the poor power efficiency and bulky size due to the free-space dispersive line and the need to couple light into fiber twice.

For MPM endoscopy, femtosecond fiber laser is promising since it is directly fiber connected, compact, not susceptible to alignment issues, and less costly, in comparison to a Ti:Sapphire laser. Two types of fiber lasers, Yb-doped fiber laser operating at $1 \mu \mathrm{m}$ wavelength and Er-doped fiber (EDF) laser operating at $1.55 \mu \mathrm{m}$, have emerged as promising excitation sources for MPM endoscopy. ${ }^{20-22}$ Two compact MPM endoscopy systems were reported using $\mathrm{Yb}$-doped fiber lasers, in which a grating pair was used before the MPM handheld probe for precompensating the dispersion to achieve a pulsewidth of $125 \mathrm{fs}^{20}$ and sub$500 \mathrm{fs},{ }^{21}$ respectively. Murari et al. ${ }^{22}$ reported a fiber-optic MPM endoscopy with a customized EDF laser, where a 700-m-long SMF was used to compress the laser pulse from 290 to $\sim 300$ fs. Due to the relatively long excitation wavelengths of the fiber lasers, they cannot efficiently excite intrinsic TPEF signal from native tissue constituents, such as nicotinamide adenine dinucleotide hydrogen and flavin adenine dinucleotide. Fluorescence staining is usually required to generate TPEF contrast. ${ }^{23}$ Instead of TPEF, Horton et al. ${ }^{24}$ used an EDF laser at $1.7 \mu \mathrm{m}$ for exciting intrinsic three-photon fluorescence imaging in vivo. However, the excitation efficiency of three-photon excitation fluorescence is much lower than that of TPEF.

The frequency-doubled EDF laser centers around $780 \mathrm{~nm}$, which can be utilized as the excitation source for stainingfree MPM imaging. ${ }^{25-27}$ Unruh et al. ${ }^{25}$ and Chun et al. ${ }^{26}$ used commercial EDF lasers with a frequency-doubled output as the excitation source in benchtop MPM systems, where the fiber lasers were used as a free-space laser source. Recently, a miniature MPM endoscopy system was reported by Huland 
et al. ${ }^{27}$ which utilized a commercial EDF laser with a frequencydoubled output. The frequency-doubled EDF laser had a freespace output and was recoupled into a hollow core PBF for delivering 780-nm pulses. This process required precise alignment for fiber recoupling, introduced coupling loss, and lost the convenience of direct fiber connection.

A miniature fiber-optic MPM endoscopy system based on the frequency-doubled EDF laser was demonstrated in our previous work. ${ }^{9}$ The laser pulse was directly coupled via a piece of SMF to the imaging probe where the pulse was frequencydoubled in the distal end of the probe. Femtosecond pulses at 780-nm wavelength were obtained and used to excite intrinsic TPEF signal from tissues. Ex vivo MPM imaging of unstained biological tissues was demonstrated at 0.4 frames/s. However, the imaging speed was relatively low and the frequency-doubled pulse had side lobes that reduced the temporal quality of the excitation source. The imaging speed was mainly limited by the low MPM excitation efficiency. To increase the imaging speed, the MPM signal level needs to be increased by improving the MPM excitation efficiency.

In this paper, a systematic optimization of the frequencydoubled EDF laser to increase the MPM excitation efficiency is carried out. The effect of the frequency-doubling process on the MPM excitation and the factors that affect the characteristics of the frequency-doubled pulse are analyzed. Optimization on the laser source and its frequency-doubling process is also carried out experimentally. Using the optimized frequency-doubled pulse as the excitation source, MPM imaging with significantly increased speed is demonstrated by ex vivo imaging of unstained biological tissues. It is shown that the miniature MPM endoscopy with the frequency-doubled EDF laser has great potential in translating MPM imaging into clinical applications.

\section{System Optimization}

\subsection{System Configuration}

Figure 1 shows the schematic diagram of the miniature MPM endoscopy system with the frequency-doubled EDF laser. The system contains four major parts: (1) EDF oscillator, (2) bidirectionally pumped EDF amplifier, (3) frequency-doubling unit, and (4) MPM endoscopy imaging head. Details about the four parts are described below, respectively.

\section{EDF oscillator}

An EDF oscillator, mode-locked via nonlinear polarization rotation, ${ }^{28,29}$ is used as the seed laser. The oscillator is pumped by a diode laser at $980 \mathrm{~nm}$. The optical fiber section of the oscillator cavity contains 0.96-m-long EDF (EDF80, OFS) with GVD of $+0.061 \mathrm{ps}^{2} / \mathrm{m}$ at $1560 \mathrm{~nm}$. The total length of SMF (SMF-28, Corning) in the oscillator is $2.4 \mathrm{~m}$, with GVD of $-0.023 \mathrm{ps}^{2} / \mathrm{m}$ at $1560 \mathrm{~nm}$. The net dispersion of the oscillator is designed to be slightly positive by adjusting the lengths of the SMF and EDF, respectively. The net dispersion based on the fiber lengths is approximately $+0.003 \mathrm{ps}^{2}$ at $1560 \mathrm{~nm}$.

\section{Bidirectionally pumped EDF amplifier}

The fiber amplifier contains a piece of EDF which is bidirectionally pumped by two 980 -nm laser diodes with an overall

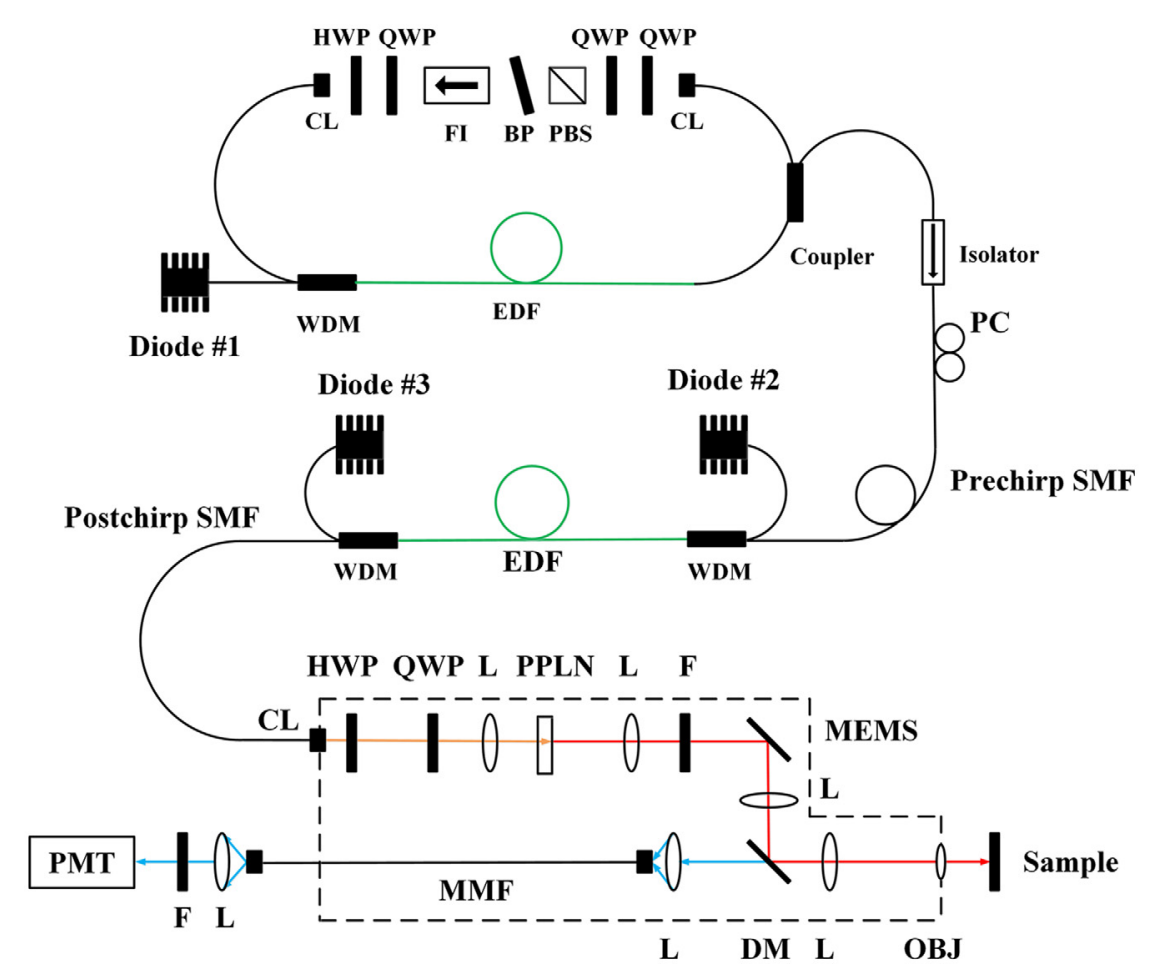

Fig. 1 Schematic of the miniature MPM endoscopy system with the frequency-doubled EDF laser. The dashed box indicates the MPM endoscopy imaging head. EDF, Er-doped fiber; HWP, half-wave plate; QWP, quarter-wave plate; BP, birefringent plate; FI, Faraday isolator; PBS, polarization beam splitter; $\mathrm{CL}$, collimating lens; WDM, wavelength division multiplexer; $\mathrm{PC}$, polarization controller; SMF, single mode fiber; M, mirror; L, lens; PPLN, periodically poled $\mathrm{MgO}$ : $\mathrm{LiNbO}_{3}$ crystal; $\mathrm{F}$, filter; MEMS, microelectromechanical scanning mirror; DM, dichroic mirror; OBJ, objective lens; MMF, multimode fiber; PMT, photomultiplier tube. 
CW-power of $1.2 \mathrm{~W}$. The seed pulse from the oscillator first propagates through a section of prechirp fiber, mostly composed by SMF. After the prechirp fiber, the pulse enters a 1.65-m-long EDF. Finally, the amplified pulse is delivered via a pigtailed collimator with a piece of postchirp SMF. The postchirp SMF has anomalous dispersion at $1560 \mathrm{~nm}$ and thus it compensates the dispersion and compresses the pulse after the EDF. The amplified output power is around $260 \mathrm{~mW}$.

\section{Frequency-doubling unit}

Frequency-doubling is achieved through a periodically poled $\mathrm{MgO}: \mathrm{LiNbO}_{3}$ (PPLN) crystal (MSHG1550-0.5-0.3, Covesion Ltd.). A half-wave plate and a quarter-wave plate are used to adjust the polarization for efficient frequency-doubling in the PPLN. The fundamental beam is focused into the PPLN by an aspheric lens with a focal length of $7.5 \mathrm{~mm}$ (A375TM-C, $f=7.5 \mathrm{~mm}, \mathrm{NA}=0.3$, Thorlabs). Another aspheric lens (A397TM-B, $f=11.0 \mathrm{~mm}, \mathrm{NA}=0.3$, Thorlabs) is used to collimate the frequency-doubled beam and a filter (RG9, Schott) is used to block the residual fundamental power.

\section{MPM endoscopy imaging head}

The dashed box in Fig. 1 indicates the MPM endoscopy imaging head, which includes the frequency-doubling unit. Therefore, there is no need to recouple the light into fiber again after the frequency-doubling. After the frequency-doubling unit, the beam is directed to a 2.4-mm-diameter twodimensional microelectromechanical scanning (MEMS) mirror (Mirrorcle Tech., Inc.) to raster scan the beam. Two identical $\varnothing 1 / 2$ in. achromatic doublets (AC127-019-A, $f=19.0 \mathrm{~mm}$, Thorlabs) are used to relay the beam from the MEMS scanner to a miniature objective lens. An aspheric lens C150TME-A ( $f=2.0 \mathrm{~mm}, \mathrm{NA}=0.50$, Thorlabs) is used as the objective lens. The emitted MPM signals are collected in the backward direction and separated from the excitation light by a dichroic mirror (FF670-SDi01, Semrock). The MPM signals are coupled into a multimode fiber (MMF) patch cable $(\varnothing 1500 \mu \mathrm{m}$, $\mathrm{NA}=0.39$, Thorlabs) and sent to a photomultiplier tube (PMT) (H9305-03, Hamamatsu). A blocking filter with a passband from 350 to $650 \mathrm{~nm}$ (FF01-680/SP-25, Semrock) is placed in front of the PMT to remove the residual of the backreflected excitation light. Since the EDF laser is directly coupled into the imaging head through a standard SMF and the emitted MPM signal is directed to the PMT by an MMF, the MPM endoscopy system is compact and fiber connected. The field-of-view of the MPM system is $160 \mu \mathrm{m} \times 250 \mu \mathrm{m}$ (328 pixels $\times 512$ pixels). The lateral resolution is $1.2 \mu \mathrm{m}$ and the axial resolution is $16 \mu \mathrm{m}$.

\subsection{MPM Intensity}

To optimize the system, the influence of the excitation laser properties on the MPM intensity is analyzed. The timeaveraged TPEF intensity excited by femtosecond pulse can be described as ${ }^{30}$

$$
\left\langle I_{\mathrm{TPEF}}\right\rangle \cong \delta_{2} \eta \frac{P_{\mathrm{ave}}^{2}}{\tau_{p} f_{p}}\left(\frac{N A^{2}}{h c \lambda}\right)^{2}
$$

where, $\tau_{p}$ is the pulse duration, $P_{\text {ave }}$ is the average laser power, $f_{p}$ is the repetition rate of the laser, $\delta_{2}$ is the two-photon absorption cross-section, $\eta$ is the quantum yield, NA is the numerical aperture of the objective lens, $\lambda$ is the excitation wavelength, and $h$ and $c$ are Planck's constant and the speed of light in vacuum, respectively. As we can see, the TPEF intensity depends quadratically on the average laser power and inversely on the pulse duration and repetition rate of the excitation light. The SHG intensity has a similar dependence. Increasing the average laser power can increase the MPM intensity. Nevertheless, the laser power needs to be kept below the safety limit in order not to cause tissue damage and loss of cell viability. ${ }^{31}$ MPM intensity can also be increased by shortening the pulse duration. In this study, we will improve the MPM intensity by optimizing the EDF laser system.

\subsection{Frequency-Doubling by PPLN}

To increase the MPM intensity, the frequency-doubled pulse needs to be optimized for shorter pulsewidth and higher average power within the laser safety limit. The design considerations on frequency-doubling by PPLN will be discussed. The frequencydoubling process depends on the input pulsewidth, bandwidth, and phase matching. The related theory is explained below.

In the classical theory of SHG in a nonlinear crystal, assuming plane-wave and nondepleted pump, the electric field of the frequency-doubled output is described as ${ }^{32}$

$\tilde{E}_{2 \omega}(L)=\frac{-j \omega_{0} d_{\mathrm{eff}}}{n_{2} c} \tilde{E}_{\omega}^{2}(0) L \exp (j \Delta k L / 2) \operatorname{sinc}(\Delta k L / 2)$,

where $L$ is the crystal length, $d_{\text {eff }}$ is the nonlinear coefficient, and $n_{2}$ is the refractive index associated with the frequencydoubled frequency $2 \omega_{0}$. The difference in the propagation constants of the fundamental and frequency double beams, $\Delta k=k_{2}-2 k_{1}$, is the phase mismatch. The conversion from the fundamental beam to the frequency-doubled beam is most efficient when the phase mismatch is zero.

When there is phase mismatch as $\Delta k \neq 0$, the power is only efficiently converted from the fundamental beam to the frequency-doubled beam within the coherent buildup length $l_{c}=\pi / \Delta k$. On the other hand, PPLN relies on quasi-phasematching (QPM) to increase the interaction length and conversion efficiency. In QPM, the sign of $d_{\text {eff }}$ alternates periodically with a period $\Lambda$. Thus, a structural periodicity is built into the nonlinear crystal, to repeatedly correct the relative phase between the $\omega_{0}$ and $2 \omega_{0}$ fields. This periodic modulation on the nonlinear coefficient acts as a grating, which introduces a momentum and modifies the phase matching parameter in first-order QPM as ${ }^{32}$

$\Delta k^{\prime}=\Delta k+\frac{2 \pi}{\Lambda}$

With the modified phase matching parameter, the electric field of the frequency-doubled output in QPM is given as ${ }^{32}$

$\tilde{E}_{2 \omega}(L)=\frac{-j \omega_{0} d_{\mathrm{eff}}}{n_{2} c} \tilde{E}_{\omega}^{2}(0) L G \exp \left(j \Delta k^{\prime} L / 2\right) \operatorname{sinc}\left(\Delta k^{\prime} L / 2\right)$.

Comparing Eqs. (2) and (4), $\Delta k$ is replaced by $\Delta k^{\prime}$ in QPM, where the momentum from the grating assists the phase matching condition. Although QPM can increase the interaction 
length between the fundamental and frequency-doubled beams, the conversion efficiency is lower than that in a single crystal with perfect phase matching. This efficiency reduction is described by the factor $G=2 / \pi$. $^{32}$

From the electric field, intensity can be calculated by $I_{(i)}=\frac{1}{2} \varepsilon_{0} c n_{(i)}\left|\tilde{E}_{(i)}\right|^{2}$, where $\varepsilon_{0}$ is the permittivity of free space. ${ }^{32}$ The subscript $i$ stands for either $\omega$ or $2 \omega$. For frequency-doubling of femtosecond pulse in a thin PPLN, the time dependence of intensity is introduced and quasicontinuous-wave limit is applied. Assuming phase matching as $\Delta k^{\prime}=0$, a time-averaged intensity for pulsed excitation can be obtained as

$$
\left\langle I_{2 \omega}(t)\right\rangle=G^{2} \Gamma^{2} g\left\langle I_{\omega}(t)\right\rangle^{2} L^{2},
$$

where $\Gamma^{2}=\frac{2 \omega_{0}^{2} d_{\text {eff }}^{2}}{\varepsilon_{0} c^{3} n_{1}^{2} n_{2}}\left(n_{1}\right.$ is the refractive index associated with the fundamental frequency $\omega_{0}$ ), and $g=\left\langle I_{\omega}^{2}(t)\right\rangle /\left\langle I_{\omega}(t)\right\rangle^{2}$ is a measure of the second-order temporal coherence of the excitation source. For a repetitively pulsed laser, $g$ can be expressed as $g=g_{p} / \tau_{p} f_{p}$, where $g_{p}$ is a dimensionless quantity. For a Gaussian pulse, ${ }^{33} g_{p}=0.664$.

To increase the conversion efficiency, the laser beam is focused into the PPLN. The averaged power of the fundamental and frequency-doubled beams can be calculated by integrating the averaged intensity over the beam cross section ${ }^{32}$

$$
P_{(i)}=\left\langle I_{(i)}(t)\right\rangle \int \mathrm{d} A\left|u_{(i)}\right|^{2},
$$

where $u_{(i)}$ is the corresponding Gaussian beam function. By combining Eqs. (5) and (6), the averaged power of the frequency-doubled beam is obtained as ${ }^{32}$

$$
P_{2 \omega}=\frac{g_{p}}{\tau_{p} f_{p}} G^{2} \Gamma^{2}\left\langle I_{\omega}(t)\right\rangle^{2} L^{2} \int \mathrm{d} A\left|u_{2 \omega}\right|^{2}=\frac{g_{p} G^{2} \Gamma^{2} P_{\omega}^{2} L^{2}}{\tau_{p} f_{p} A_{\mathrm{eff}}},
$$

where $A_{\text {eff }}=\frac{\left(\int \mathrm{d} A\left|u_{\omega}\right|^{2}\right)^{2}}{\int \mathrm{d} A\left|u_{2 \omega}\right|^{2}}$. For the fundamental and frequencydoubled beams, $\left|u_{2 \omega}\right|=\left|u_{\omega}\right|^{2}$ can be applied. For a Gaussian beam, $A_{\text {eff }}=\pi w_{0(\omega)}^{2}$, where $w_{0(\omega)}$ is the beam waist of the fundamental beam. Optimum focusing is obtained when the depth of focus $b \cong L,{ }^{32}$ where $b=2 n_{1} \pi w_{0(\omega)}^{2} / \lambda$. Thus, the output power of the frequency-doubled beam can be obtained as

$P_{2 \omega}=\frac{2 n_{1} g_{p} L G^{2} \Gamma^{2} P_{\omega}^{2}}{\lambda \tau_{p} f_{p}}$.

As we can see in Eq. (8), the output power of the frequencydoubled beam in PPLN depends linearly on the PPLN length and inversely on the input pulsewidth under the perfect phase matching condition.

Nevertheless, the perfect phase matching condition $\Delta k^{\prime}=0$ in QPM is only achieved at a specific wavelength. For a femtosecond pulse with a broad bandwidth, an acceptance bandwidth is defined for a particular length of PPLN, where phase matching is approximately achieved within the acceptance bandwidth. The acceptance bandwidth is determined by the dispersion property of the crystal. For a Gaussian pulse, it is given as ${ }^{34}$

$\delta \lambda=\frac{0.4429 \lambda}{L}\left|\frac{n_{2}-n_{1}}{\lambda}+\frac{\partial n_{1}}{\partial \lambda}-\frac{1}{2} \frac{\partial n_{2}}{\partial \lambda}\right|^{-1}$.
It shows that the acceptance bandwidth is inversely dependent on the crystal length $L$. For shorter PPLN length, its acceptance bandwidth $\delta \lambda$ is broader. In order to efficiently convert the input pulse into frequency-doubled pulse, the bandwidth of the input fundamental beam $\Delta \lambda$ should match with the acceptance bandwidth of the PPLN so that $\Delta \lambda=\delta \lambda$. For transform-limited pulse, the product of the bandwidth and pulsewidth is a constant. For a Gaussian pulse, the transform-limited pulsewidth $\tau_{p}=0.4429 \lambda^{2} / c \delta \lambda$ can be calculated from Eq. (9) as

$\tau_{p}=\frac{\lambda L}{c}\left|\frac{n_{2}-n_{1}}{\lambda}+\frac{\partial n_{1}}{\partial \lambda}-\frac{1}{2} \frac{\partial n_{2}}{\partial \lambda}\right|$.

Therefore, the pulsewidth is linearly proportional to the PPLN length as limited by its acceptance bandwidth. Substituting Eq. (10) into Eq. (8), we get

$P_{2 \omega} \cong \frac{2 n_{1} c g_{p} G^{2} \Gamma^{2} P_{\omega}^{2}}{f_{p} \lambda^{2}}\left|\frac{n_{2}-n_{1}}{\lambda}+\frac{\partial n_{1}}{\partial \lambda}-\frac{1}{2} \frac{\partial n_{2}}{\partial \lambda}\right|^{-1}$.

When the full-width-half-maximum (FWHM) bandwidth of the fundamental beam matches the acceptance bandwidth of the PPLN, the power of the frequency-doubled output is irrelevant to the PPLN length. In the quasicontinuous-wave limit, the pulsewidth of the frequency-doubled pulse equals $\tau_{p} / \sqrt{2}$ for a Gaussian beam, where $\tau_{p}$ is the fundamental pulsewidth. ${ }^{29}$

Figure 2 shows the theoretically transform-limited pulsewidth as a function of the PPLN length. The solid line and dashed line represent how the transform-limited pulsewidth of the fundamental beam and the frequency-doubled beam, respectively, change with the PPLN length, which both show a linear relationship.

From Eq. (1), the MPM intensity depends quadratically on the average power and inversely on the pulsewidth of the excitation laser beam. Here, the frequency-doubled beam is used as the excitation source for the MPM imaging. When the spectral bandwidth of the fundamental beam matches with the acceptance bandwidth of the PPLN, the average power of the frequency-doubled beam does not depend on the PPLN length but the pulsewidth of the frequency-doubled beam depends linearly on the PPLN length. Therefore, the relationship between the time-averaged MPM intensity and the PPLN length $L$ is simply $\left\langle I_{\mathrm{MPM}}\right\rangle \propto \frac{1}{L}$. The MPM intensity versus the PPLN length is also plotted in Fig. 2 as the dash-dotted line.

In principle, the MPM intensity can be increased by choosing a shorter PPLN length, which can provide a shorter frequencydoubled pulsewidth without affecting its output power. However, there are practical challenges when the PPLN length is very short due to the high nonlinear effects at very short pulsewidth. For example, a 0.1-mm-long PPLN has an acceptance bandwidth of $123 \mathrm{~nm}$ at $1560 \mathrm{~nm}$ and a corresponding transform-limited pulsewidth of 29 fs. At 29-fs pulsewidth, the peak power will be extremely high and the fiber nonlinearity will be significant. It will also be challenging to compensate the dispersion and find components that can cover this broad bandwidth. Considering the trade-off between the advantages of short-pulse and the practical challenges, a PPLN length of $0.3 \mathrm{~mm}$ is selected as the optimum design under the current experimental condition. It has an acceptance bandwidth of $42 \mathrm{~nm}$ at $1560 \mathrm{~nm}$ and a transform-limited fundamental pulsewidth of 87 fs. In our previous study, ${ }^{9}$ a 1-mm-long PPLN was used, which had an acceptance bandwidth of $12 \mathrm{~nm}$ at $1560 \mathrm{~nm}$ 


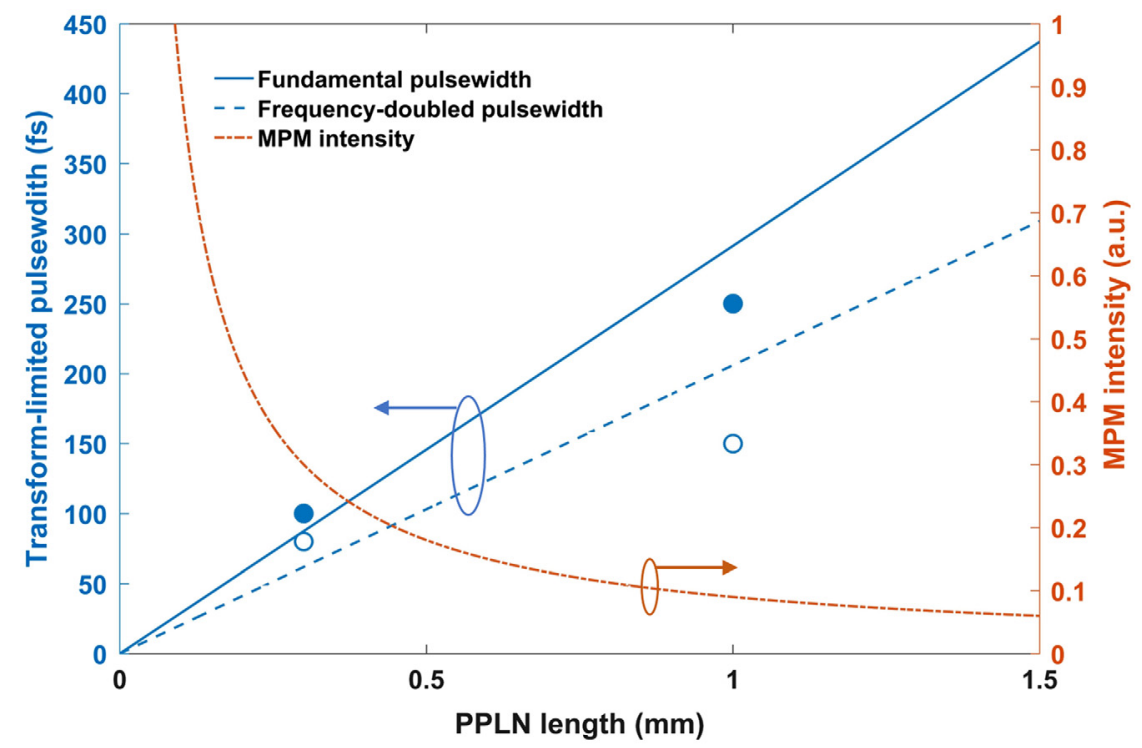

Fig. 2 Dependence of the pulsewidth and MPM intensity on the PPLN length. The experimentally measured pulsewidths at PPLN length 0.3 and $1.0 \mathrm{~mm}$ are marked by the solid circles for the fundamental beam and open circles for the frequency-doubled beam. The ellipses with arrows indicate the corresponding $Y$-axis of the curves.

and a transform-limited fundamental pulsewidth of 291 fs. Compared to the 1-mm-long PPLN, the 0.3-mm-long PPLN can potentially reduce the pulsewidth to $1 / 3$ of the pulsewidth of the 1-mm-long PPLN.

\subsection{Er-Doped Fiber Laser Optimization}

The design consideration on the fiber laser oscillator and the amplifier is to achieve a clean spectrum that matches the acceptance bandwidth of the PPLN and obtain a nearly transformlimited pulsewidth. For the selected 0.3-mm-long PPLN, the targeted FWHM bandwidth of the EDF laser should be $42 \mathrm{~nm}$. Figure 3 shows the spectrum and the intensity autocorrelation of the oscillator measured at the reflection port of the polarization beam splitter (PBS). The central wavelength is $\sim 1560 \mathrm{~nm}$, the FWHM spectral bandwidth is $47 \mathrm{~nm}$, and the $-10-\mathrm{dB}$ spectral bandwidth is $77 \mathrm{~nm}$. The FWHM spectral bandwidth of the oscillator is close to the acceptance bandwidth of the $0.3-\mathrm{mm}$ long PPLN. The pulsewidth is $247 \mathrm{fs}$, assuming a Gaussian pulse. Here, the pulse is not compressed and is slightly chirped. The average power of the oscillator through the output port of the coupler is $4 \mathrm{~mW}$ and the repetition rate is $47 \mathrm{MHz}$.

The seed pulse from the oscillator is then sent to the fiber amplifier which boosts the output power to $260 \mathrm{~mW}$. At this power level, the interplay between GVD and SPM is significant, which determines the characteristics of the amplified pulse. Figure 4 compares the results obtained at several prechirp fiber lengths. The spectrum and intensity autocorrelation of the amplified fundamental pulse and the spectrum of the frequency-doubled pulse after PPLN are shown. The fundamental pulsewidth is measured at the output of the postchirp SMF before pulse compression is applied. Gaussian pulse shape is assumed for all the pulsewidth calculation in this paper. Since the output spectrum is not smooth and contains multiple
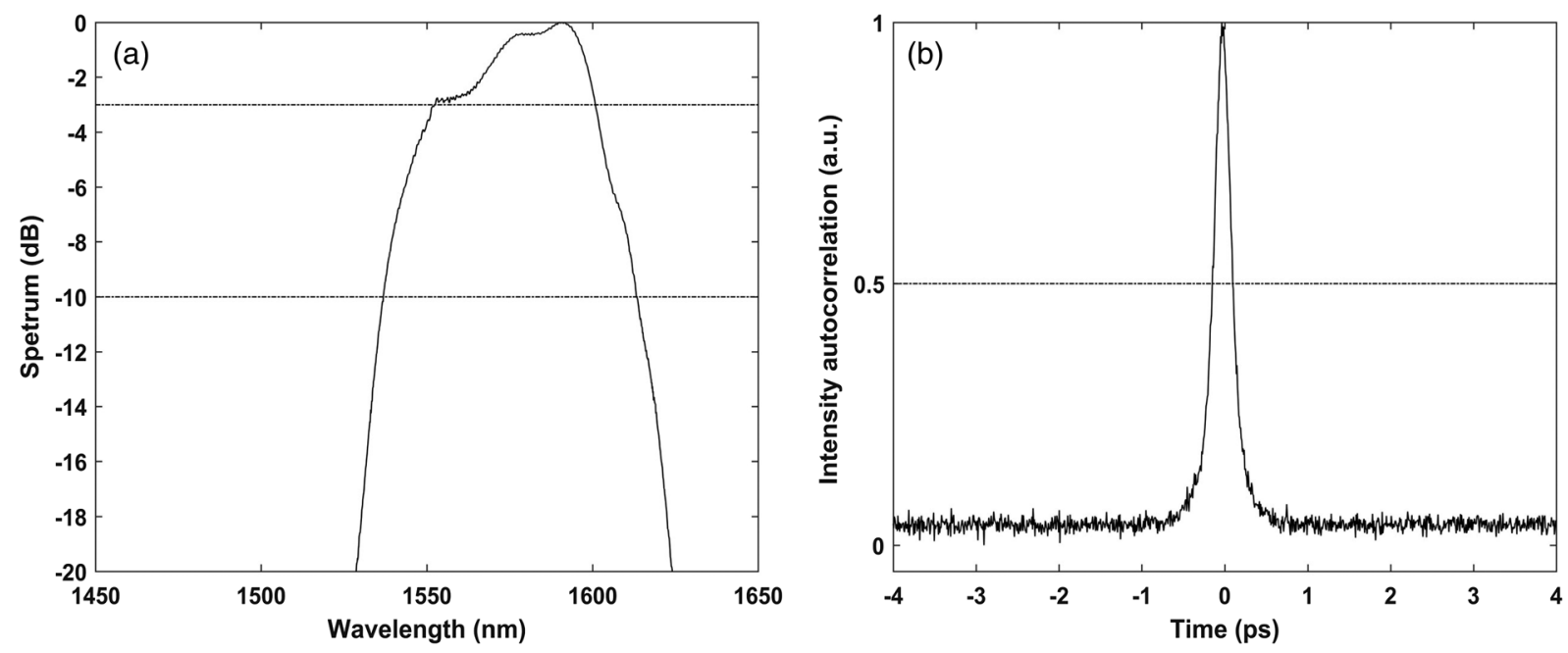

Fig. 3 (a) The spectrum and (b) the intensity autocorrelation of the oscillator output. 
Pre-chirp fiber

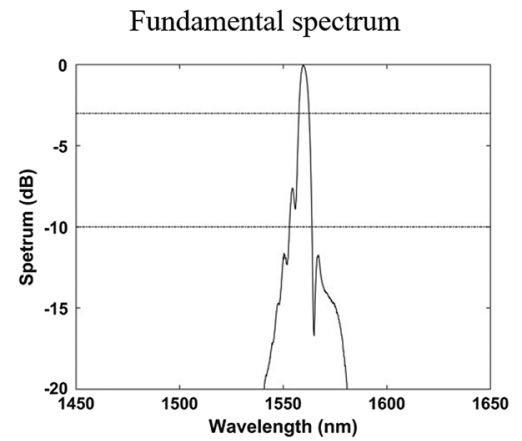

(a) $\mathrm{SMF}=12.8 \mathrm{~m}$ $\left(-0.30 \mathrm{ps}^{2}\right)$.

(b) $\mathrm{SMF}=6.3 \mathrm{~m}$ $\left(-0.14 \mathrm{ps}^{2}\right)$.

(c) $\mathrm{SMF}=3.5 \mathrm{~m}$ $\left(-0.08 \mathrm{ps}^{2}\right)$.
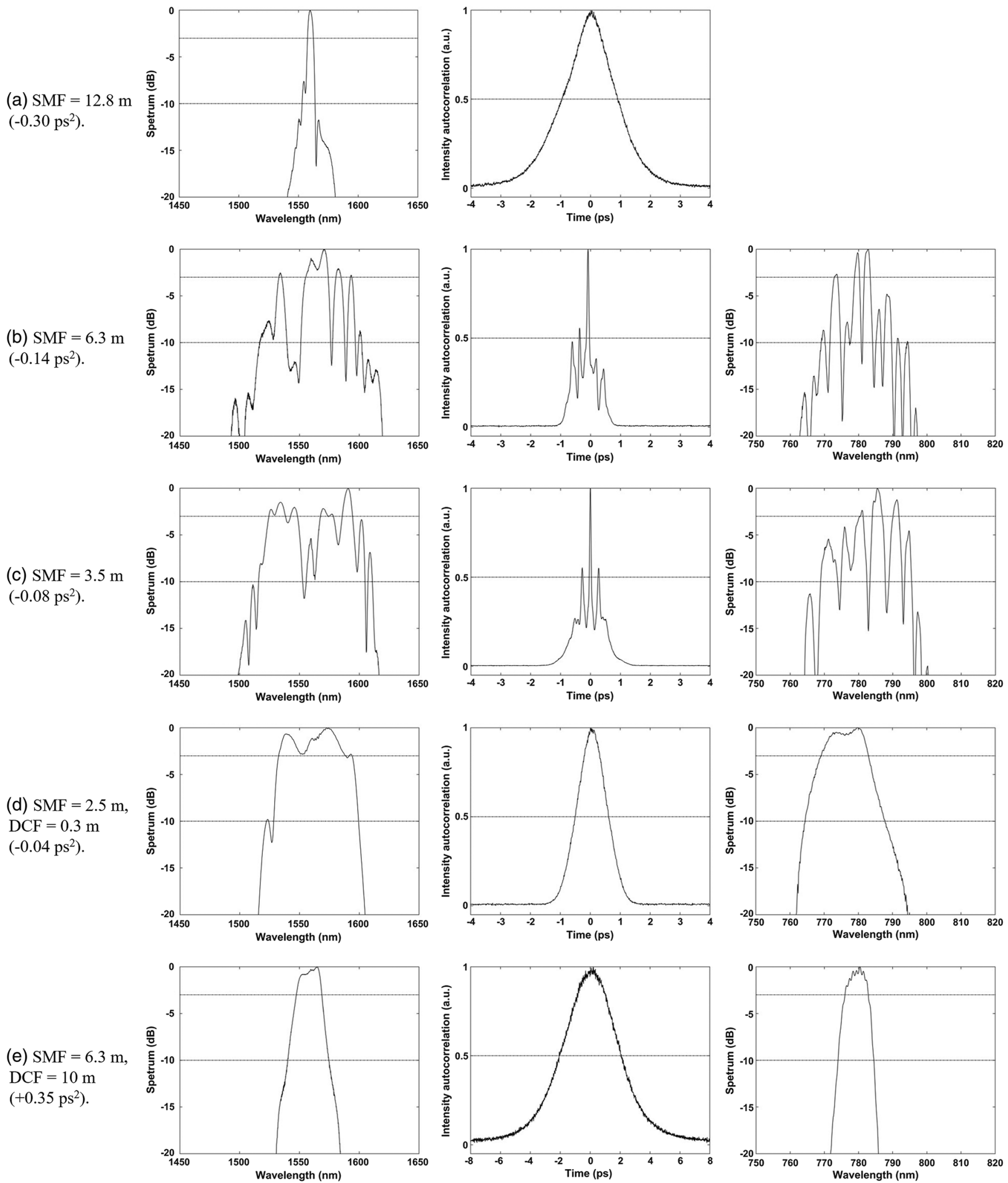

Fig. 4 The spectra and intensity autocorrelation traces of the fundamental beam, and the corresponding spectra of the frequency-doubled beam, at different prechirp fiber lengths. (a) SMF $=12.8 \mathrm{~m}$. (b) $S M F=6.3 \mathrm{~m}$. (c) $\mathrm{SMF}=3.5 \mathrm{~m}$. (d) $\mathrm{SMF}=2.5 \mathrm{~m}$ and $\mathrm{DCF}=0.3 \mathrm{~m}$. (e) $\mathrm{SMF}=6.3 \mathrm{~m}$ and $\mathrm{DCF}=10 \mathrm{~m}$. 
peaks for some cases, $-10-\mathrm{dB}$ spectral bandwidth is used for comparison.

Figure 4(a) shows the case when the prechirp SMF is $12.8 \mathrm{~m}$, corresponding to a prechirp dispersion of $-0.30 \mathrm{ps}^{2}$. The postchirp SMF fiber is $0.9 \mathrm{~m}$. It shows a narrowed spectrum with the $-10-\mathrm{dB}$ spectral bandwidth of $11 \mathrm{~nm}$, and a broad pulsewidth of 1.9 ps. Since the spectral bandwidth is too narrow, no frequencydoubling is performed.

Figure 4(b) shows the case when the prechirp SMF is $6.3 \mathrm{~m}$, corresponding to a prechirp dispersion of $-0.14 \mathrm{ps}^{2}$. The postchirp SMF is reduced to $0.25 \mathrm{~m}$ from now on. The spectrum contains multiple peaks, and the $-10-\mathrm{dB}$ spectral bandwidth is $66 \mathrm{~nm}$. Pulse break-up occurs and multiple peaks appear in the autocorrelation trace. The pulsewidth is estimated as 346 fs. The spectrum of the frequency-doubled beam also has multiple peaks and the $-10-\mathrm{dB}$ spectral bandwidth is about $21 \mathrm{~nm}$. Because of pulse breakup, the fundamental pulse cannot be compressed to transform-limited pulsewidth.

Figure 4(c) is the case when the prechirp SMF is $3.5 \mathrm{~m}$, corresponding to a prechirp dispersion of $-0.08 \mathrm{ps}^{2}$. The $-10-\mathrm{dB}$ spectral bandwidth of the fundamental beam is $90 \mathrm{~nm}$ and pulse breakup also exists. The pulsewidth is estimated as $328 \mathrm{fs}$. The spectrum of the frequency-doubled beam has multiple peaks and the $-10-\mathrm{dB}$ spectral bandwidth is about $27 \mathrm{~nm}$.

Figure 4(d) is the case when the prechirp fiber includes $2.5-\mathrm{m}$ SMF and 0.3-m dispersion compensating fiber (DCF), corresponding to the prechirp dispersion of $-0.04 \mathrm{ps}^{2}$. DCF (DCF38, Thorlabs) with GVD of $+0.049 \mathrm{ps}^{2} / \mathrm{m}$ at $1560 \mathrm{~nm}$ is used to introduce positive dispersion in the prechirp part, because the SMF in the fiber-based components (such as isolator and coupler) cannot be too short in practice. The spectrum of the fundamental beam, in this case, is cleaner compared to the previous cases. The $-10-\mathrm{dB}$ spectral bandwidth is $82 \mathrm{~nm}$ and the FWHM spectral bandwidth is $53 \mathrm{~nm}$, which is close to the acceptance bandwidth of the 0.3-mm-long PPLN. The pulsewidth of the fundamental beam is $1.1 \mathrm{ps}$. No pulse breakup is observed. The corresponding spectrum of the frequencydoubled beam is relatively smooth and the $-10-\mathrm{dB}$ spectral bandwidth is $25 \mathrm{~nm}$. This is the optimal case for the $0.3-\mathrm{mm}$ long PPLN because it has no pulse breakup and has a clean spectrum that is close to the acceptance bandwidth of the PPLN.

Figure 4(e) shows the case when the prechirp fiber includes 6.3-m SMF and 10-m DCF, corresponding to the prechirp dispersion of $+0.35 \mathrm{ps}^{2}$. The -10 - $\mathrm{dB}$ spectral bandwidth is narrowed to $34 \mathrm{~nm}$. This spectrum is too narrow to fully use the PPLN. The amplified pulse has a broad pulsewidth of 4.1 ps. The $-10-\mathrm{dB}$ spectral bandwidth of the frequency-doubled beam is $11 \mathrm{~nm}$.

Figure 5 summarizes the relationship between the prechirp dispersion and the properties of the amplified fundamental pulse and frequency-doubled pulse, respectively. The spectral bandwidth (circle) and pulsewidth (triangle) of the amplified fundamental pulse, and the spectral bandwidth of the frequency-doubled pulse (cross) are plotted as a function of the prechirp dispersion, which is calculated based on the lengths of the prechirp SMF and DCF. Two different postchirp SMF lengths are used in the experiment. For prechirp $\leq-0.20 \mathrm{ps}^{2}$, the postchirp SMF is $0.9 \mathrm{~m}$ (data marked by open symbols). For prechirp $\geq-0.20 \mathrm{ps}^{2}$, the postchirp SMF is $0.25 \mathrm{~m}$ (data marked by solid symbols). To investigate the effect of prechirp fiber on the amplified pulse, it is desirable to minimize the nonlinear broadening from the postchirp fiber by shortening the postchirp SMF.

In the EDF amplifier, the interplay between GVD and SPM determines the characteristics of the amplified pulse. For an initially negatively chirped pulse (acquired from the prechirp $\mathrm{SMF}$ ), the negative chirp will be gradually compensated as the pulse propagates in the EDF. Thus, the pulse is compressed to reach a minimal pulsewidth when the prechirp is fully compensated, and afterward the pulse broadens again as it propagates in the remaining EDF. SPM-induced spectral broadening is significant near the zero chirp position where minimal

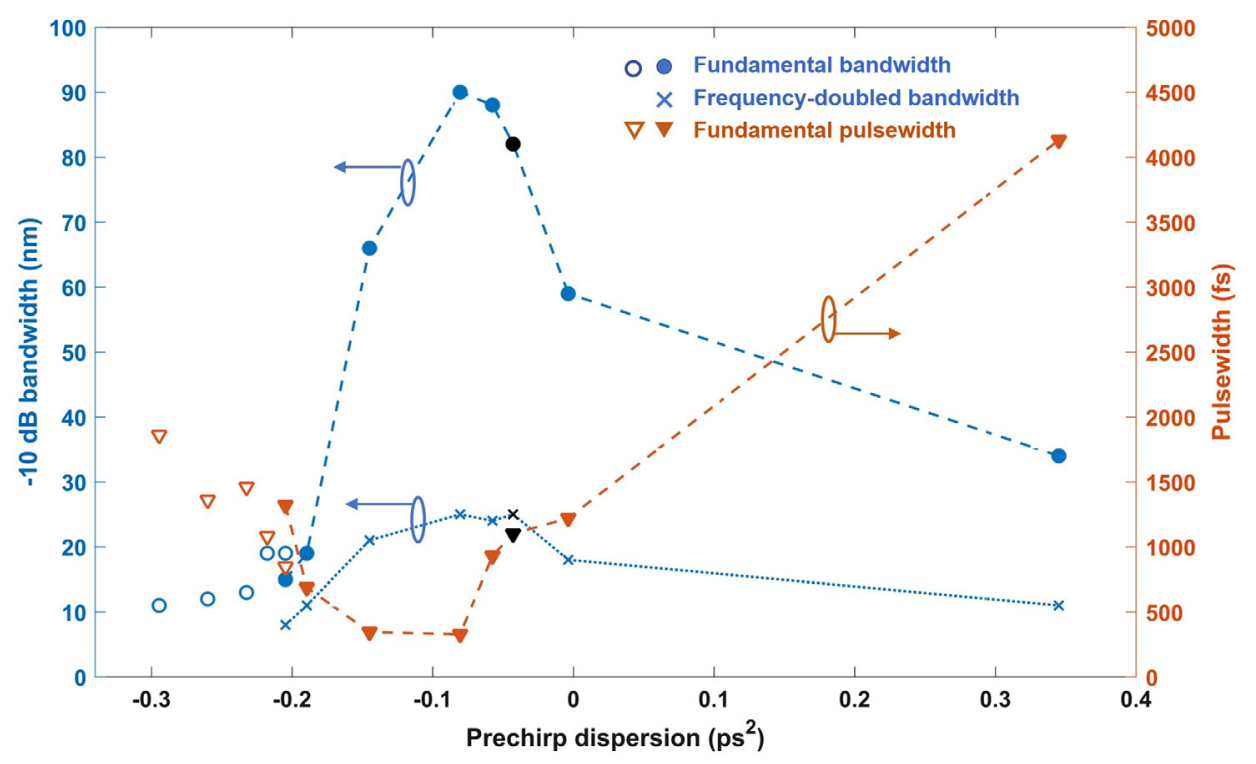

Fig. 5 The spectral bandwidth (circle) and pulsewidth (triangle) of the amplified fundamental pulse, and the spectral bandwidth of the frequency-doubled pulse (cross), at different prechirp dispersions. Solid symbols represent the data points with postchirp $S M F=0.25 \mathrm{~m}$, and open symbols represent the data points with postchirp SMF $=0.9 \mathrm{~m}$. The black symbols indicate the optimum case. The ellipses with arrows indicate the corresponding $Y$-axis of the curves. 
pulsewidth is reached. Adjusting the prechirp fiber length can vary the position where the minimal pulsewidth occurs and thus controls the spectrum and pulsewidth of the amplified pulse. Several regions can be shown in Fig. 5.

When the prechirp dispersion is largely negative $\left(<-0.2 \mathrm{ps}^{2}\right)$, the amount of negative prechirp dispersion is much larger than the amount of positive dispersion in the EDF. The amplified pulse has broad pulsewidth (in the ps regime) during the passage through the entire EDF, and thus GVD dominates over SPM. ${ }^{35}$ As a result, the spectral bandwidth of the amplified pulse is reduced due to gain narrowing and the pulsewidth is largely stretched.

When the prechirp dispersion is less negative $(-0.2$ to $-0.08 \mathrm{ps}^{2}$ ), the effect of SPM increases, which induces significant nonlinear phase shift. The fundamental spectrum becomes very broad and highly structured. In this regime, with $1.2-\mathrm{W}$ pump power in total, the nonlinear phase shift becomes extremely high and pulse breakup occurs. ${ }^{36}$

When the prechirp dispersion is slightly negative $(-0.08$ to $0 \mathrm{ps}^{2}$ ), the minimal pulsewidth occurs in the first half of the EDF where the power is low or moderate. Thus, the nonlinear phase shift is reduced and the amplified pulse has a spectrum with minimum structures, and pulse breakup is eliminated. This regime is close to parabolic amplification..$^{37,38}$ This is the optimal operating region of the fiber amplifier, and this case is highlighted by the black markers in Fig. 5 .

When the prechirp dispersion is in the largely positive domain, it is in the parabolic amplification regime. ${ }^{37}$ The pulsewidth is stretched into ps due to positive chirp, and thus GVD dominates over SPM. ${ }^{35}$ The spectral bandwidth is significantly reduced due to gain narrowing. For all the cases, the bandwidth of the frequency-doubled beam shows a similar trend as the bandwidth of the fundamental beam.

\subsection{Pulse Compression}

Based on the investigation above, the optimal prechirp is found for 2.5-m-long SMF plus 0.3-m-long DCF. The corresponding spectra for the fundamental and frequency-doubled beams are shown in Fig. 4(d). The FWHM spectral bandwidth of the fundamental beam is $53 \mathrm{~nm}$, which is close to the acceptance bandwidth of the 0.3-mm-long PPLN (42-nm FWHM). The amplified fundamental pulse is positively chirped with a broad pulsewidth of $1.1 \mathrm{ps}$. Dispersion compensation is needed in order to compress the pulse to near transform limited.

At $1560 \mathrm{~nm}$, SMF has anomalous GVD, which can be used to compress the fundamental pulse coming from the EDF amplifier. This is achieved by optimizing the length of the postchirp SMF. Figure 6 shows the FWHM pulsewidth and $-10-d B$ spectral bandwidth of the fundamental pulse at the output of the postchirp SMF with different lengths. As the length increases from 0.8 to $1.0 \mathrm{~m}$, the pulsewidth is sharply reduced as the initial positive chirp gained in the EDF amplifier is compensated. When the length increases from 1.0 to $2.0 \mathrm{~m}$, the FWHM pulsewidth of the autocorrelation trace does not change much. However, the output pulse displays multiple side peaks. The shortest pulsewidth of the central peak in the autocorrelation trace is found at the postchirp fiber length of 1.3 to $1.4 \mathrm{~m}$, where the side peaks are also relatively small. On the other hand, the spectral bandwidth has an overall increasing trend due to increased SPM when the postchirp SMF length increases from 0.8 to $2.0 \mathrm{~m}$. The dip in the spectral bandwidth at $1.3 \mathrm{~m}$ is caused by measurement inaccuracy when the spectrum curve is highly structured with multiple peaks. The optimum postchirp fiber length is where the pulsewidth is compressed to minimum while the SPM induced spectral broadening is still moderate. Considering both the pulsewidth and spectral bandwidth, the optimal postchirp SMF length is found to be in the range of 1.3 to $1.4 \mathrm{~m}$.

The compression result when the postchirp SMF is $1.4 \mathrm{~m}$ is shown in Figs. 7(a)-7(d). Figure 7(a) is the intensity autocorrelation of the fundamental pulse after SMF compression, and it shows the FWHM pulsewidth of $\sim 80 \mathrm{fs}$. Nevertheless, the autocorrelation trace also shows strong side peaks. The spectrum of the fundamental pulse after SMF compression is shown in Fig. 7(b) and the $-10-\mathrm{dB}$ bandwidth is $118 \mathrm{~nm}$. Compared with the spectrum before SMF compression, the SPM introduced by the postchirp SMF broadens the fundamental spectrum

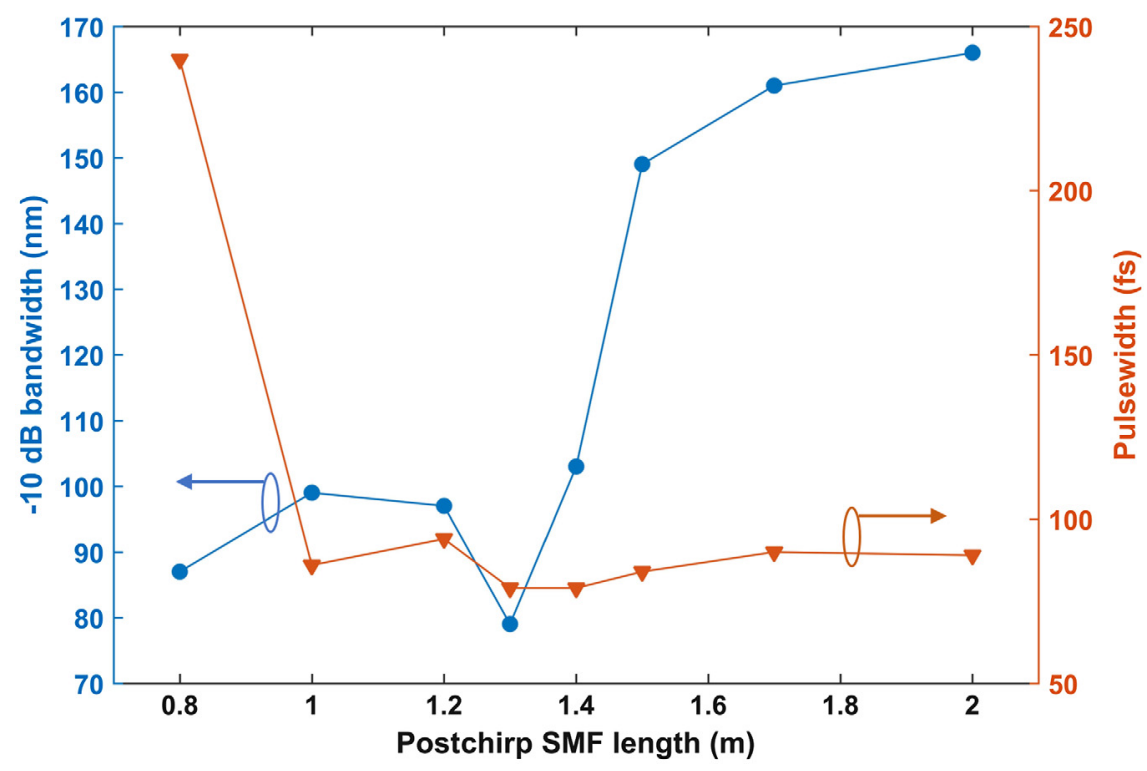

Fig. 6 The effect of postchirp SMF length on the FWHM pulsewidth (triangle) and -10-dB bandwidth (circle) of the fundamental pulse at the postchirp SMF output. The ellipses with arrows indicate the corresponding $Y$-axis of the curves. 

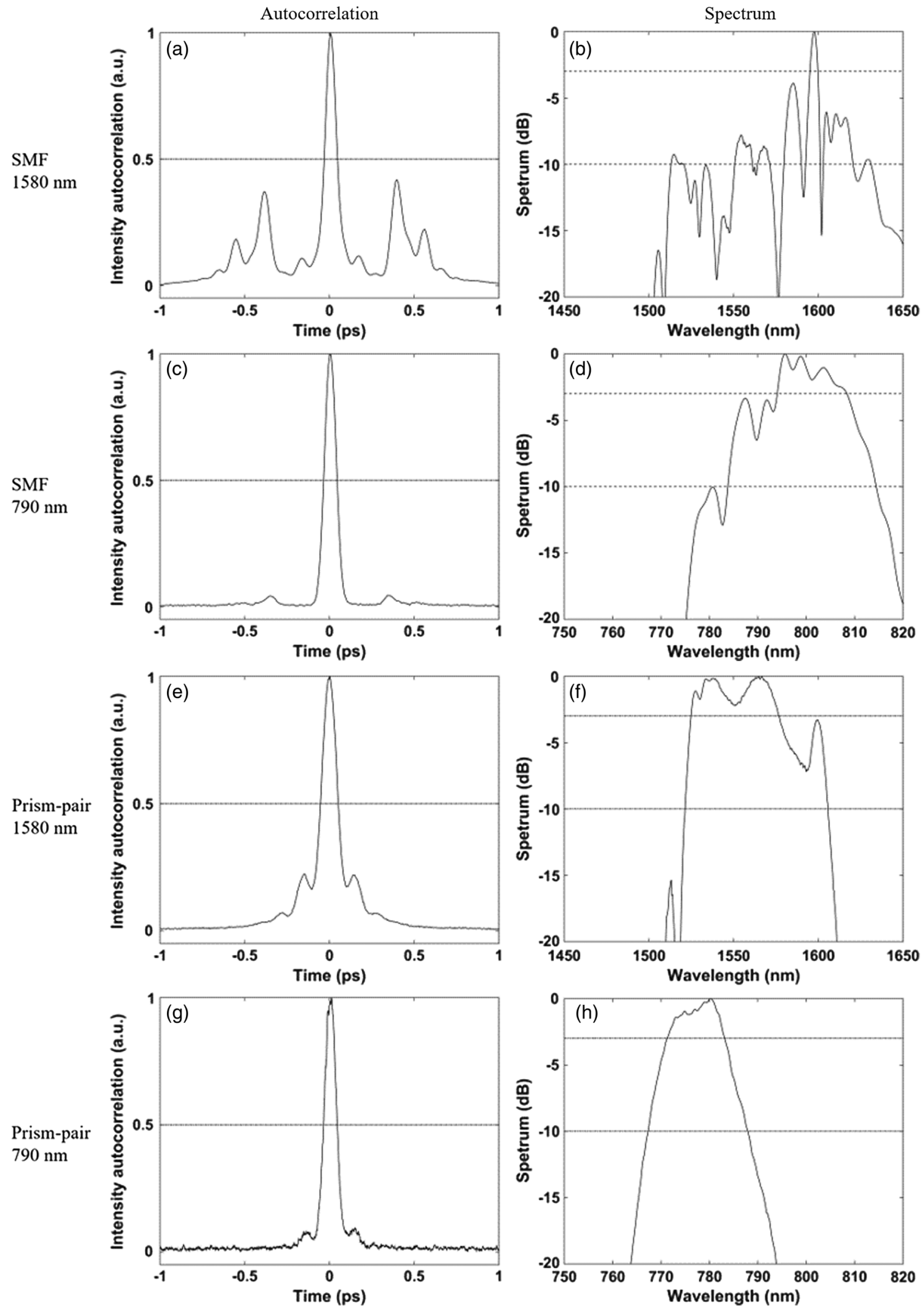

Fig. 7 Comparisons of the pulse compression results by SMF and silicon prism pair. (a)-(d) The spectra and intensity autocorrelation traces of the fundamental beam and the frequency-doubled beam after SMF compression. (e)-(h) The spectra and intensity autocorrelation traces of the fundamental beam and the frequency-doubled beam after prism-pair compression. 
Table 1 Summary of pulse characteristics obtained by the $0.3-$ and $1-\mathrm{mm}$ PPLN. ${ }^{9}$

\begin{tabular}{|c|c|c|c|c|c|c|c|c|}
\hline \multirow{3}{*}{$\begin{array}{l}\text { PPLN } \\
\text { length } \\
(\mathrm{mm}) \\
\end{array}$} & \multirow{3}{*}{$\begin{array}{c}\text { Acceptance } \\
\text { bandwidth } \\
(\mathrm{nm})\end{array}$} & \multirow{3}{*}{$\begin{array}{c}\text { Pulse } \\
\text { compression }\end{array}$} & \multicolumn{3}{|c|}{ Fundamental beam } & \multicolumn{2}{|c|}{ Frequency-doubled beam } & \multirow{3}{*}{$\begin{array}{c}\text { Power } \\
\text { conversior } \\
\text { efficiency }\end{array}$} \\
\hline & & & \multicolumn{2}{|c|}{ Bandwidth (nm) } & \multirow{2}{*}{$\begin{array}{l}\text { Pulsewidth } \\
\text { (fs) }\end{array}$} & \multirow{2}{*}{$\begin{array}{c}\text { FWHM } \\
\text { bandwidth } \\
(\mathrm{nm})\end{array}$} & \multirow{2}{*}{$\begin{array}{l}\text { Pulsewidth } \\
\text { (fs) }\end{array}$} & \\
\hline & & & FWHM & $-10 \mathrm{~dB}$ & & & & \\
\hline \multirow[b]{2}{*}{0.3} & \multirow[b]{2}{*}{42} & Silicon prism & 52 & 84 & 100 & 12 & 80 & $42 \%$ \\
\hline & & SMF & & 118 & 80 & 15 & 80 & $39 \%$ \\
\hline $1.0^{9}$ & 12 & SMF & 8 & 60 & 250 & 5 & 150 & $40 \%$ \\
\hline
\end{tabular}

and causes uneven structures. The central wavelength is slightly shifted to a longer wavelength. The intensity autocorrelation of the corresponding frequency-doubled pulse is shown in Fig. 7(c) and it shows the FWHM pulsewidth of $80 \mathrm{fs}$. A relatively clean autocorrelation trace with minimal side peaks is observed. The spectrum of the frequency-doubled pulse is shown in Fig. 7(d) and it shows the FWHM bandwidth of $15 \mathrm{~nm}$. Comparing the frequency-doubled pulse with the fundamental pulse, both the autocorrelation trace and the spectrum are much cleaner without complicate structures. This is likely due to the limited acceptance bandwidth of the PPLN, which limits the bandwidth of the frequency-doubled pulse and cleans its pulse shape. The frequency-doubled pulse has a time-bandwidth-product of 0.59 , which is nearly transform-limited. With SMF as the pulse compressor, effective pulse compression is achieved. For 170-mW laser power right before the PPLN, 66-mW output power of the frequency-doubled beam is obtained, corresponding to the power conversion efficiency of $39 \%$.

To compare the pulse quality after SMF compression with the compression method of free-space optics, the pulse is also compressed by a silicon prism pair (Crystran). The intensity autocorrelation and spectrum of the compressed fundamental pulse and the corresponding frequency-doubled pulse are shown in Figs. 7(e)-7(h). The pulsewidth of the compressed fundamental pulse and frequency-doubled pulse are 100 and $80 \mathrm{fs}$, respectively. The FWHM bandwidth of the compressed fundamental pulse and frequency-doubled pulse are 52 and $12 \mathrm{~nm}$, respectively. The spectrum of the compressed fundamental pulse is similar to the pulse spectrum before compression. For $150-\mathrm{mW}$ laser power right before the PPLN, 60-mW output power of the frequency-doubled beam is obtained, corresponding to the power conversion efficiency of $42 \%$.

Comparing the pulse compression by SMF versus prism pair, SMF introduces a significant amount of nonlinearity due to SPM, and the prism-pair compression is almost linear with no additional nonlinearity. Nevertheless, as the frequencydoubling only happens within the acceptance bandwidth of the PPLN, the frequency-doubled pulse can still achieve a similar pulsewidth and bandwidth as the results achieved by prism pair. Furthermore, using SMF compression, the EDF laser and the MPM endoscopy imaging head can be connected directly via the postchirp SMF, which makes the whole system highly compact and portable. In our SMF compression and delivery of femtosecond intense pulses at $1560-\mathrm{nm}$ wavelength, anomalous dispersion from SMF can compensate the normal dispersion from EDF, which enables all-fiber-based dispersion control and direct fiber connection between the fiber laser and the MPM endoscopy. Femtosecond pulse can be directly delivered to the distal end of the MPM endoscopy using standard SMF. This femtosecond pulse delivery scheme is simple and has high power efficiency. By frequency doubling the pulse from 1560 to $780 \mathrm{~nm}$ at the distal end of the imaging head, it can excite intrinsic TPEF and SHG signals in tissues label-free.

Table 1 summarizes the characteristics of the fundamental and frequency-doubled pulses using the 0.3-mm-long PPLN and 1-mm-long PPLN. ${ }^{9}$ The pulsewidth of the frequencydoubled pulse is reduced from 150 to 80 fs when the PPLN length is reduced from 1 to $0.3 \mathrm{~mm}$. However, the conversion efficiency is nearly equal. The similar conversion efficiency is consistent with our theoretical analysis that the output power of the PPLN does not depend on the PPLN length. Since the MPM intensity is inversely proportional to the excitation pulsewidth, the MPM intensity is expected to increase by two to three times when the PPLN length is reduced from 1 to $0.3 \mathrm{~mm}$, under the same average power. The experimental pulsewidth of the fundamental and frequency-doubled pulses using the 0.3- and 1-mm-long PPLN, ${ }^{9}$ respectively, are also compared with the theoretical results in Fig. 2 (marked by solid and open circles for the fundamental pulse and the frequency-doubled pulse, respectively). For the 0.3 -mm-long PPLN, the measured pulsewidth matches reasonably well with the theoretical estimation. In our previous study using the 1-mm-long PPLN in Ref. 9 shown as the last row in Table 1, both the fundamental and frequency-doubled pulses had large side peaks. The measured pulsewidths only considered the main peak. Therefore, the measured pulsewidths were underestimations of the actual pulsewidths.

\section{MPM Imaging}

The optimized fiber laser system is used as the excitation source for the miniature MPM endoscopy system shown in Fig. 1. The system is applied to image ex vivo, unprocessed (unstained and unfixed) biological tissue samples. With shorter pulse, higher MPM excitation efficiency can be obtained, which is critical for MPM imaging of native signals in tissues. With higher MPM excitation efficiency, the pixel dwell time can be reduced and the imaging speed can be increased, correspondingly. To test this, four different pixel dwell times of 10, 5, 2 , and $1 \mu \mathrm{s} /$ pixel, which corresponds to four different frame rates of $0.4,0.8,2$, and 4 frames/s, are applied to image the tissue samples. The MPM images of the mouse (CD-1 strain) skin and kidney are shown in Fig. 8. The average excitation power at the sample is about $40 \mathrm{~mW}$. Figures $8(\mathrm{a})-8(\mathrm{~d})$ show the MPM images of the dermal layer in the murine skin, in which the hair follicle and collagen fibers can be distinguished clearly. Figures 8(e)-8(h) show the MPM images of intracellular 

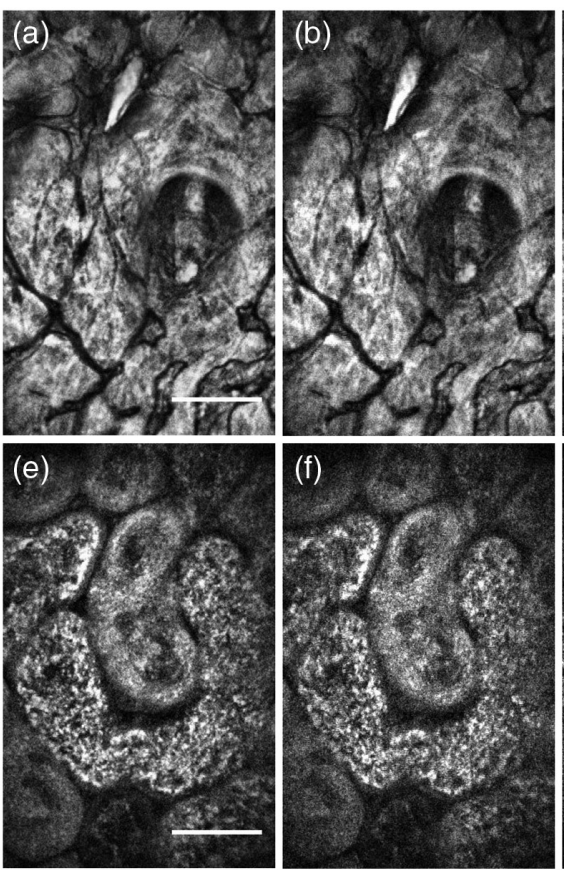
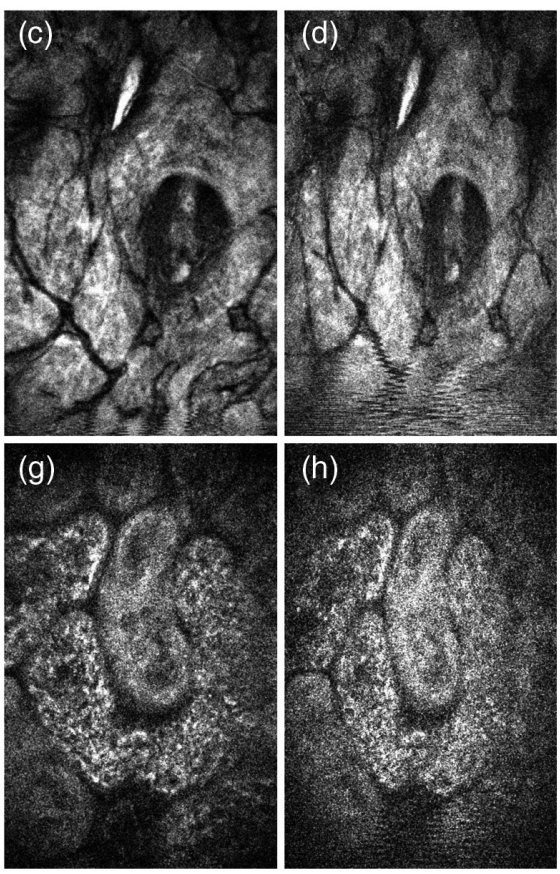

Fig. 8 (a)-(d) MPM images of the murine skin at 0.4, 0.8, 2, and 4 frames/s, respectively. (e)-(h) MPM images of the murine kidney at $0.4,0.8,2$, and 4 frames/s, respectively. Scale bar is $50 \mu \mathrm{m}$.

flavins of epithelial cells of the murine kidney tubules, ${ }^{15}$ in which the epithelial cells can be clearly seen.

When the pixel dwell time is reduced from 10 to $1 \mu \mathrm{s} / \mathrm{pixel}$, there are still reasonable MPM signals to show the tissue structures. In our previous work on MPM endoscopy with 1-mmlong PPLN, ${ }^{9}$ the imaging speed is limited to 0.4 frames/s $(10 \mu \mathrm{s} / \mathrm{pixel})$ due to the relatively low MPM excitation efficiency. After optimizing the laser system to reduce the pulsewidth and improve the temporal quality of the pulse, the imaging speed is significantly increased by almost 10 times and an imaging speed of 4 frames/s is achieved. As the scanning speed of the MEMS mirror is increased to $1 \mu \mathrm{s} /$ pixel (4 frames/s), distortion induced by the MEMS scanner appears near the bottom of the image. The scanning range in the horizontal axis (fast axis) is also slightly increased as the scanning frequency gets closer to the resonance frequency. The distortion is caused by the limited scanning speed of the current MEMS mirror. In the future, in order to achieve real-time imaging, MEMS scanner with a higher scanning speed will be required.

In this paper, the reported system optimization is on the laser source and particularly shortening the pulsewidth. As the pulsewidth gets shorter, the nonlinear effect becomes more significant. Thus, other optimization approaches also need to be considered to further increase the imaging speed. Improving the focusing quality of the miniature objective lens will be an important approach. Objective lens with higher NA and lower distortion can potentially reduce the spot size and thus increase the MPM excitation efficiency and imaging speed furthermore.

\section{Conclusions}

Developing miniature MPM endoscopy that can be used for clinical applications requires a compact laser source and fast imaging speed. The frequency-doubled EDF laser is shown to be a great choice for such a purpose. To increase the imaging speed, system optimization is carried out by reducing the pulsewidth of the excitation source. It is found that the pulsewidth of the frequency-doubled output linearly depends on the PPLN length while the output power is irrelevant with the PPLN length. By choosing a 0.3-mm thickness of the PPLN and matching the bandwidth of the fundamental beam with the acceptance bandwidth of the PPLN, frequency-doubled pulse with the pulsewidth of $80 \mathrm{fs}$ is achieved. Through the systematic optimization, high MPM excitation efficiency is obtained and imaging speed at 4 frames/s is demonstrated on ex vivo imaging of unstained biological tissues. This speed is 10 times faster than our previous reported study. Furthermore, pulse compression with SMF is achieved, which makes the system highly compact. The results show that miniature MPM endoscopy with the frequency-doubled EDF laser can potentially translate MPM imaging technology for clinical applications.

\section{Disclosures}

The authors have no financial interests or potential conflict of interest to disclose concerning this work.

\section{Acknowledgments}

We would like to thank Professor David J. Jones and Dr. Arthur K. Mills at the University of British Columbia (UBC) for their tremendous help and discussion. We would also like to thank the Centre for Disease Modeling and Animal Care Services at UBC for providing the animal tissue samples. This project was supported by Canadian Institutes of Health Research (CIHR)/Collaborative Health Research Projects (CPG151974), Natural Sciences and Engineering Research Council of Canada (NSERC)/Collaborative Health Research Projects (CHRP 508405-17), and NSERC/Discovery Grants Program (RGPIN-2017-05913). 


\section{References}

1. P. T. So et al., "Two-photon excitation fluorescence microscopy," Ann. Rev. Biomed. Eng. 2, 399-429 (2000).

2. M. C. Skala et al., "Multiphoton microscopy of endogenous fluorescence differentiates normal, precancerous, and cancerous squamous epithelial tissues," Cancer Res. 65, 1180-1186 (2005).

3. W. R. Zipfel, R. M. Williams, and W. W. Webb, "Nonlinear magic: multiphoton microscopy in the biosciences," Nat. Biotechnol. 21, 13691377 (2003).

4. P. J. Campagnola and L. M. Loew, "Second-harmonic imaging microscopy for visualizing biomolecular arrays in cells, tissues and organisms," Nat. Biotechnol. 21, 1356-1360 (2003).

5. F. Helmchen and W. Denk, "Deep tissue two-photon microscopy," Nat. Methods 2, 932-940 (2005).

6. F. Helmchen et al., "A miniature head-mounted two-photon microscope: high-resolution brain imaging in freely moving animals," Neuron 31, 903-912 (2001).

7. Y. Wu et al., "Scanning all-fiber-optic endomicroscopy system for 3D nonlinear optical imaging of biological tissues," Opt. Express 17, 79077915 (2009).

8. D. M. Huland et al., "In vivo imaging of unstained tissues using long gradient index lens multiphoton endoscopic systems," Biomed. Opt. Express 3, 1077-1085 (2012).

9. L. Huang et al., "Miniature fiber-optic multiphoton microscopy system using frequency-doubled femtosecond Er-doped fiber laser," Biomed. Opt. Express 7, 1948-1956 (2016).

10. R. L. Harzic et al., "Rigid and high-numerical-aperture two-photon fluorescence endoscope," Appl. Opt. 48, 3396-3400 (2009).

11. W. Piyawattanametha et al., "In vivo brain imaging using a portable $2.9 \mathrm{~g}$ two-photon microscope based on a microelectromechanical systems scanning mirror," Opt. Lett. 34, 2309-2311 (2009).

12. Y. Zhao, H. Nakamura, and R. J. Gordon, "Development of a versatile two-photon endoscope for biological imaging," Biomed. Opt. Express 1, 1159-1172 (2010).

13. D. R. Rivera et al., "Compact and flexible raster scanning multiphoton endoscope capable of imaging unstained tissue," Proc. Natl. Acad. Sci. U. S. A. 108, 17598-17603 (2011).

14. Y. Zhang et al., "A compact fiber-optic SHG scanning endomicroscope and its application to visualize cervical remodeling during pregnancy," Proc. Natl. Acad. Sci. U. S. A. 109, 12878-12883 (2012).

15. G. Ducourthial et al., "Development of a real-time flexible multiphoton microendoscope for label-free imaging in a live animal," Sci. Rep. 5, 18303 (2015)

16. W. Liang et al., "Nonlinear optical endomicroscopy for label-free functional histology in vivo," Light Sci. Appl. 6, e17082 (2017).

17. S. Clark, F. Ilday, and F. Wise, "Fiber delivery of femtosecond pulses from a Ti:sapphire laser," Opt. Lett. 26, 1320-1322 (2001).

18. M. Lelek et al., "Coherent femtosecond pulse shaping for the optimization of a non-linear micro-endoscope," Opt. Express 15, 10154-10162 (2007).

19. W. Liang, G. Hall, and X. Li, "Spectro-temporal dispersion management of femtosecond pulses for fiber-optic two-photon endomicroscopy," Opt. Express 26, 22877-22893 (2018).

20. G. Liu et al., "Multiphoton microscopy system with a compact fiberbased femtosecond-pulse laser and handheld probe," J. Biophotonics 4, 34-39 (2011).

21. Á. Krolopp et al., "Handheld nonlinear microscope system comprising a $2 \mathrm{MHz}$ repetition rate, mode-locked $\mathrm{Yb}$-fiber laser for in vivo biomedical imaging," Biomed. Opt. Express 7, 3531-3542 (2016).
22. K. Murari et al., "Compensation-free, all-fiber-optic, two-photon endomicroscopy at $1.55 \mu \mathrm{m}$," Opt. Lett. 36, 1299-1301 (2011).

23. W. R. Zipfel et al., "Live tissue intrinsic emission microscopy using multiphoton-excited native fluorescence and second harmonic generation," Proc. Natl. Acad. Sci. U. S. A. 100, 7075-7080 (2003).

24. N. G. Horton et al., "In vivo three-photon microscopy of subcortical structures within an intact mouse brain," Nat. Photonics 7, 205-209 (2013).

25. J. R. Unruh et al., "Two-photon microscopy with wavelength switchable fiber laser excitation," Opt. Express 14, 9825-9831 (2006).

26. W. Chun, D. Do, and D.-G. Gweon, "Design and demonstration of multimodal optical scanning microscopy for confocal and two-photon imaging," Rev. Sci. Instrum. 84, 013701 (2013).

27. D. M. Huland et al., "Multiphoton gradient index endoscopy for evaluation of diseased human prostatic tissue ex vivo," J. Biomed. Opt. 19, 116011 (2014).

28. M. Hofer et al., "Mode locking with cross-phase and self-phase modulation," Opt. Lett. 16, 502-504 (1991).

29. K. Tamura et al., "Soliton versus nonsoliton operation of fiber ring lasers," Appl. Phys. Lett. 64, 149-151 (1994).

30. A. Diaspro, G. Chirico, and M. Collini, "Two-photon fluorescence excitation and related techniques in biological microscopy," Q. Rev. Biophys. 38, 97-166 (2005).

31. S. Tang et al., "Effect of pulse duration on two-photon excited fluorescence and second harmonic generation in nonlinear optical microscopy," J. Biomed. Opt. 11, 020501 (2006).

32. A. M. Weiner, Ultrafast Optics, Wiley, New Jersey (2009).

33. C. Xu and W. W. Webb, "Measurement of two-photon excitation cross sections of molecular fluorophores with data from 690 to $1050 \mathrm{~nm}$," $J$. Opt. Soc. Am. B 13, 481-491 (1996).

34. M. M. Fejer et al., "Quasi-phase-matched second harmonic generation: tuning and tolerances," IEEE J. Quantum Electron. 28, 2631-2654 (1992).

35. F. Tauser, A. Leitenstorfer, and W. Zinth, "Amplified femtosecond pulses from an Er:fiber system: nonlinear pulse shortening and self-referencing detection of the carrier-envelope phase evolution," Opt. Express 11, 594-600 (2003).

36. G. Agrawal, Nonlinear Fiber Optics, Elsevier Science, New York (2010).

37. M. Fermann et al., "Self-similar propagation and amplification of parabolic pulses in optical fibers," Phys. Rev. Lett. 84, 6010-6013 (2000).

38. J. W. Nicholson et al., "High power, single mode, all-fiber source of femtosecond pulses at $1550 \mathrm{~nm}$ and its use in supercontinuum generation," Opt. Express 12, 3025-3034 (2004).

Lin Huang is currently a PhD candidate in the Department of Electrical and Computer Engineering, University of British Columbia. Her research interest is in miniature multiphoton systems and devices, and femtosecond fiber lasers.

Shuo Tang is an associate professor in the Department of Electrical and Computer Engineering, University of British Columbia. Her research interest is in biomedical optical imaging systems and devices, including multiphoton microscopy, optical coherence tomography, and micro-endoscopy systems for biomedical applications.

Xin Zhou is currently a PhD candidate in the Department of Electrical and Computer Engineering, University of British Columbia. His research interest is in multiphoton microscopy and polarization sensitive optical coherence tomography. 